\title{
Fiber-laser-based, green-pumped, picosecond optical parametric oscillator using fan-out grating PPKTP
}

\author{
S. Chaitanya Kumar, ${ }^{1,{ }^{*}}$ S. Parsa, ${ }^{1}$ M. Ebrahim-Zadeh ${ }^{1,2}$ \\ ${ }^{1}$ ICFO-Institut de Ciencies Fotoniques, Barcelona Institute of Science and Technology, 08860 Castelldefels (Barcelona), Spain \\ ${ }^{2}$ Institucio Catalana de Recerca i Estudis Avancats (ICREA), Passeig Lluis Companys 23, Barcelona 08010, Spain \\ *Corresponding author: chaitanya.suddapalli@icfo.es
}

Received XX Month XXXX; revised XX Month, XXXX; accepted XX Month XXXX; posted XX Month XXXX (Doc. ID XXXXX); published XX Month XXXX

We report a stable, Yb-fiber-laser-based, green-pumped, picosecond optical parametric oscillator (OPO) for the near-infrared based on the nonlinear crystal, PPKTP, using fan-out grating design, and operating near room temperature. The OPO is continuously tunable across $726-955 \mathrm{~nm}$ in the signal and $1201-1998 \mathrm{~nm}$ in the idler, resulting in a total signal plus idler wavelength coverage of $1026 \mathrm{~nm}$ by grating tuning at a fixed temperature. The device generates up to $580 \mathrm{~mW}$ of average power in the signal at $765 \mathrm{~nm}$ and $300 \mathrm{~mW}$ in the idler at $1338 \mathrm{~nm}$ with an overall extraction efficiency of up to $52 \%$ and a pump depletion $>76 \%$. The extracted signal at $765 \mathrm{~nm}$ and idler at $1746 \mathrm{~nm}$ exhibit excellent passive power stability better than $0.5 \%$ and $0.8 \%$ rms, respectively, over 1 hour, in good beam quality with TEMoo mode profile. The output signal pulses have Gaussian temporal duration of 13.2 ps with a FWHM spectral bandwidth of $3.4 \mathrm{~nm}$ at $79.5 \mathrm{MHz}$ repetition rate. Power scaling limitations of the OPO due to the material properties of PPKTP are studied. (C) 2015 Optical Society of America

OCIS codes: (190.4360) Nonlinear optics, devices; (190.7110) Ultrafast nonlinear optics; (190.4400) Nonlinear optics, materials; (190.4970) Parametric oscillators and amplifiers.

http://dx.doi.org/10.1364/OL.99.099999

Rapid and continuously tunable, high-power, picosecond optical parametric oscillators (OPOs) in the near-infrared wavelength range are highly desirable for the advancement of microscopic techniques such as Coherent Anti-Stokes Raman Scattering (CARS), enabling several applications in biology and medicine [1-3]. Such OPOs are typically pumped in the green, which in turn rely on second harmonic generation (SHG) of mode-locked Nd-based solid-state or Yb-based fiber lasers [4]. Although picosecond green sources can provide high average powers, the ability to power scale green-pumped picosecond OPOs together with rapid tuning has been challenging [5]. While power scaling in OPOs depends on the available pump power and optimization of the output coupling [6], rapid tuning relies on the properties of the nonlinear material itself [7]. Several near-infrared OPOs based on birefringent nonlinear crystals such as $\mathrm{LiB}_{3} \mathrm{O}_{5}$ (LBO) [2] and $\mathrm{KTiOPO}_{4}$ (KTP) $[8,9]$, and quasi-phase-matched nonlinear materials such as periodically-poled KTP (PPKTP) [3], MgO:SPPLT [10], and MgO:PPLN [11] have been previously demonstrated.
However, in all these reports wavelength tuning has been achieved by varying the temperature of the nonlinear crystal, which is a relatively slow process.

Here we report a stable, picosecond $\mathrm{OPO}$ based on fan-out design in PPKTP, enabling rapid grating tuning by translation of the nonlinear crystal while operating close to room temperature. The OPO, which is synchronously pumped by a mode-locked frequency-doubled Yb-fiber laser in the green, can provide continuous tuning over 726-955 nm in the signal wavelength range together with corresponding idler tuning across 1201-1998 nm. A maximum average power of $580 \mathrm{~mW}$ at 765 $\mathrm{nm}$ in the signal and $300 \mathrm{~mW}$ at $1338 \mathrm{~nm}$ in the idler, an overall extraction efficiency of up to $52 \%$, and pump depletion $>76 \%$ have been obtained. To the best of our knowledge, this is the first report of a picosecond OPO based on PPKTP using a fan-out grating design.

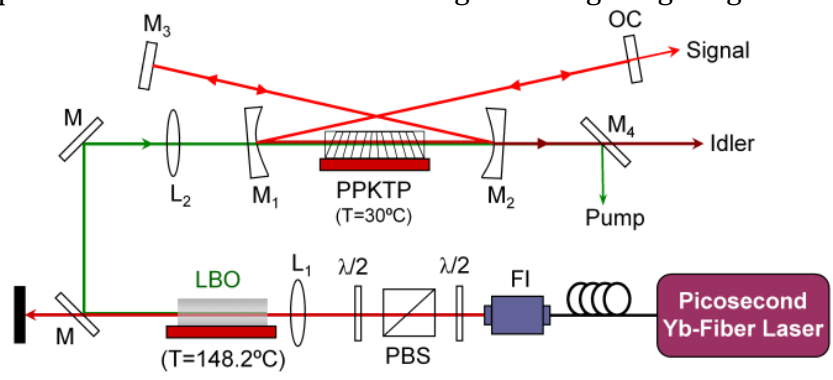

Fig. 1. Schematic experimental setup for the picosecond green-pumped PPKTP OPO. FI: Faraday isolator, $\lambda / 2$ : Half-wave plate, PBS: Polarizing beam-splitter, L: Lens, M: Mirrors, OC: Output coupler.

The schematic of the experimental setup is shown in Fig. 1. The primary pump source is a mode-locked Yb-fiber laser (Fianium, FP1060-20) delivering $\sim 20$ ps pulses at $79.5 \mathrm{MHz}$ repetition-rate, operating at a central wavelength of $1064 \mathrm{~nm}$ with a double-peak spectral bandwidth of $1.4 \mathrm{~nm}$ (FWHM). The pump light for the OPO is obtained by single-pass SHG in a 30-mm-long LBO crystal under type I $(o o \rightarrow e)$ noncritical phase-matching at a temperature of $148.2^{\circ} \mathrm{C}$. The picosecond green source provides up to $9 \mathrm{~W}$ of average power at 532 $\mathrm{nm}$ in $16.2 \mathrm{ps}$ pulses at $79.5 \mathrm{MHz}$ repetition rate with FWHM spectral bandwidth of $0.6 \mathrm{~nm}$ [12]. It exhibits passive power stability better than $0.5 \%$ rms over 16 hours in TEMoo spatial mode with beam pointing stability better than $12 \mathrm{mrad}$ over 1 hour. The nonlinear crystal for the OPO is a 30-mm-long PPKTP from Raicol Crystals with grating periods ranging from $\Lambda=9.00$ to $10.85 \mu \mathrm{m}$ in a fan-out grating design. The crystal has a 1-mm-thick, 11-mm-wide aperture, and is maintained close to the room temperature at $30^{\circ} \mathrm{C}$ for type $0(e \rightarrow e e)$ phase-matching. The crystal end-faces are antireflection (AR)-coated 
for high transmission at $532 \mathrm{~nm}$ and $720-990 \mathrm{~nm}(R<0.5 \%)$ and over $1130-2040 \mathrm{~nm}(R<5 \%)$. The green pump beam is focused to a waist radius of $w_{0} \sim 63 \mu \mathrm{m}$ at the center of the PPKTP crystal, corresponding to a focusing parameter of $\xi \sim 0.3$ [13]. The OPO is configured in a standing-wave X-cavity, with two plano-concave mirrors, $\mathrm{M}_{1}-\mathrm{M}_{2}$ ( $r=150 \mathrm{~mm}$ ), a plane mirror, $\mathrm{M}_{3}$, and a plane output coupler (OC). All mirrors are highly transmitting for the pump (T>98\%) at $532 \mathrm{~nm}$ and the idler wavelengths (T>95\%) over 1100-2100 nm, while highly reflecting $(R>99 \%)$ for the signal over $700-1000 \mathrm{~nm}$, hence ensuring singly resonant signal oscillation. A plane $\mathrm{OC}$ with varying transmission of $T \sim 31-40 \%$ over $750-965 \mathrm{~nm}$ is used to partially extract the signal power from the OPO. A dichroic mirror, $\mathrm{M}_{4}$, separates the generated idler from the transmitted pump. The total optical length of the OPO cavity is $\sim 3.77 \mathrm{~m}$, corresponding to a repetition rate of $79.5 \mathrm{MHz}$, ensuring synchronization with the pump laser repetition rate.

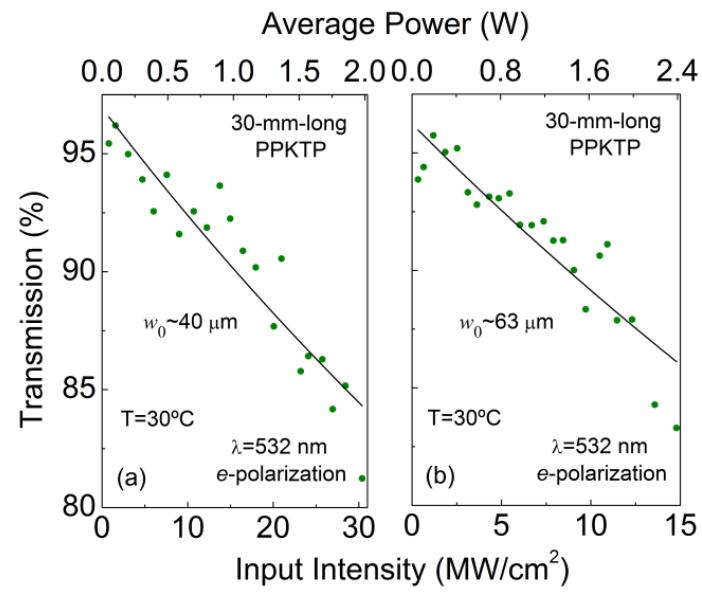

Fig. 2. Variation of the transmission of the 30-mm-long PPKTP crystal as function of input green intensity for a green beam waist radius of (a) $w_{0} \sim 40 \mu \mathrm{m}$, and (b) $w_{0} \sim 63 \mu \mathrm{m}$ in the nonlinear crystal.

Before proceeding to the characterization of the OPO, we performed transmission measurements of the 30-mm-long PPKTP crystal at an operating temperature of $30^{\circ} \mathrm{C}$. The green beam was arranged in extraordinary polarization for these measurements, as required for phase-matching in the OPO. Initially, we focused the picosecond green beam to a waist radius of $w_{0} \sim 40 \mu \mathrm{m}$ at the centre of the PPKTP crystal. The variation of the transmission as a function of the input green intensity and the corresponding average power is shown in Fig. 2(a). As the input average green power is increased from minimum up to 2 $\mathrm{W}$, corresponding to the input intensity $\sim 30 \mathrm{MW} / \mathrm{cm}^{2}$, we observe a clear drop in the transmission from $95.4 \%$ to $81.2 \%$, after correcting for the $0.5 \%$ AR coating losses at $532 \mathrm{~nm}$ on both faces of the nonlinear crystal. Using a simple model, we fitted the experimentally measured data, from which we obtained a linear absorption coefficient, $\alpha=0.01$ $\mathrm{cm}^{-1}$, and a two-photon absorption coefficient, $\beta=1.67 \mathrm{~cm} / \mathrm{GW}$ [14]. These values are significantly different from those reported earlier [14, 15], which we attribute to the use of high-repetition-rate, low-energy pulses at high average power in this work. We then relaxed the green beam waist radius to $w_{0} \sim 63 \mu \mathrm{m}$ at the centre of the PPKTP crystal and performed similar measurements, with the results shown in Fig. 2(b). However, in this case, we were not able to increase the input average green power beyond $2.4 \mathrm{~W}$, at which we observed bulk damage in the PPKTP crystal. Although this damage is not catastrophic, we were not able to use the crystal at this position any longer. It is interesting to note that the damage in this case occurred at an input green intensity of $15 \mathrm{MW} / \mathrm{cm}^{2}$, which is only $50 \%$ of the intensity used in the previous case in Fig. 2(a), where the green beam was focused to $w_{0} \sim 40$ $\mu \mathrm{m}$, thus indicating that the cause of the damage is related to the average power and not intensity. This observation also confirms that unlike the continuous-wave (cw) regime [16,17], where high $\mathrm{cw}$ powers in the green can be generated by PPKTP, in high-repetition- rate picosecond operation, power handling of the material is limited by optical damage. As such, to avoid any further damage to the crystal, we limited the input average green power to a maximum of $\sim 1.6 \mathrm{~W}$ for pumping the OPO.

In order to characterize the PPKTP picosecond OPO, we investigated the wavelength tuning performance by temperature tuning as well as grating tuning at low input pump power. For a fixed grating period of $\Lambda=9.09 \mu \mathrm{m}$, by changing the temperature of the PPKTP crystal from $30^{\circ} \mathrm{C}$ to $145^{\circ} \mathrm{C}$, were able to tune the OPO from 883 to $950 \mathrm{~nm}$ in the signal wavelength range together with the idler from 1209 to $1339 \mathrm{~nm}$, as shown in Fig 3(a). The solid and hollow circles are the experimental data, while the solid and dashed curves correspond to the theoretical
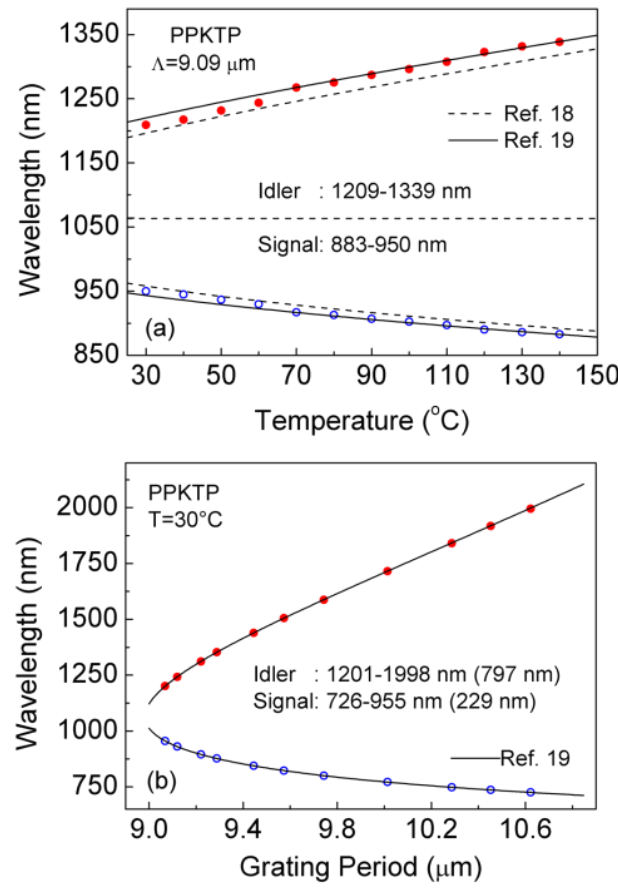

Fig. 3. (a) Temperature tuning for a fixed grating period, and (b) grating tuning for a fixed temperature of $30^{\circ} \mathrm{C}$ for the PPKTP picosecond OPO.

calculations using two different Sellemier equations from Ref. [18] and [19]. The signal wavelengths were measured using a spectrometer, while the idler wavelengths were calculated from energy conservation. As evident from Fig. 3(a), we found a better agreement between our experimental data and the calculations using Sellemier equations from Ref. [19]. We then performed grating tuning of the OPO by translating the PPKTP crystal across the pump beam while operating at a fixed temperature of $30^{\circ} \mathrm{C}$. We were able to continuously tune the OPO over $726-955 \mathrm{~nm}$ in the signal together with the idler across $1201-1998 \mathrm{~nm}$, corresponding to a total signal plus idler tuning coverage of $1026 \mathrm{~nm}$,

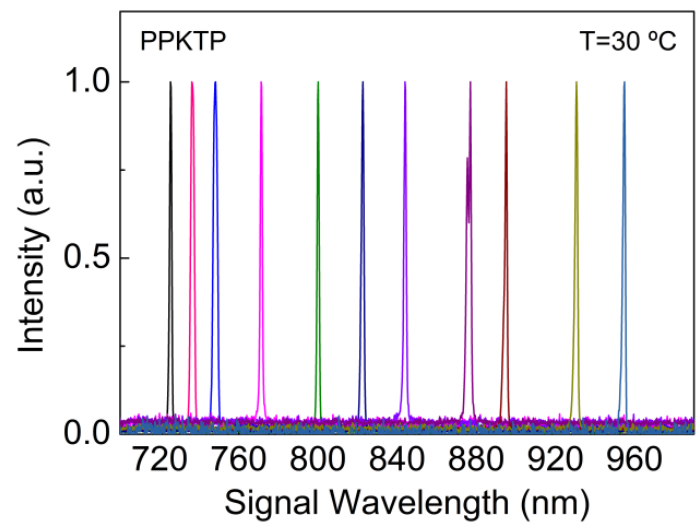

Fig. 4. Signal spectra across the tuning range of the PPKTP OPO. 
as shown in Fig. 3(b). The solid curve corresponds to the theoretical calculation using the Sellemier equations of Ref. [19], where excellent agreement with the experimental data is evident. The signal spectra obtained by translational grating tuning of the PPKTP OPO are shown in Fig. 4.

The simultaneously measured signal and idler average power, together with the overall extraction efficiency and pump depletion, across the tuning range of the PPKTP picosecond OPO is shown in Fig. 5 . For a fixed input pump power of $\sim 1.6 \mathrm{~W}$, and using an OC with varying transmission of $T \sim 31-40 \%$ over $750-965 \mathrm{~nm}$ to partially transmit the resonant signal from the cavity, we were able to extract an average signal power ranging from $540 \mathrm{~mW}$ at $750 \mathrm{~nm}$ to $460 \mathrm{~mW}$ at $964 \mathrm{~nm}$, as shown in Fig. 5(a). The corresponding idler power varied
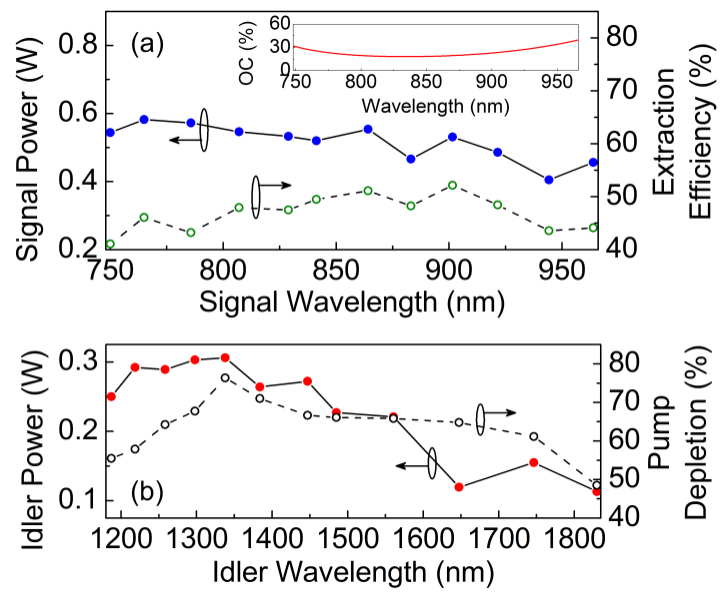

Fig. 5. (a) Signal power and extraction efficiency, (b) idler power, and pump depletion across the tuning range of the PPKTP OPO. Inset: Transmission of the OC in the signal wavelength rage.

from $250 \mathrm{~mW}$ at $1187 \mathrm{~nm}$ to $110 \mathrm{~mW}$ at $1829 \mathrm{~nm}$, with a maximum of $300 \mathrm{~mW}$ at $1338 \mathrm{~nm}$, as shown in Fig. 5(b). The OPO provided an overall extraction efficiency of up to $52 \%$ at a signal wavelength of 902 $\mathrm{nm}$, with $>40 \%$ maintained across the tuning range. A maximum pump depletion of $>76 \%$ is recorded at a generated idler wavelength of $1338 \mathrm{~nm}$, while remaining $>48 \%$ over the entire tuning range. Also shown in the inset of Fig. 5(a) is the transmission of the OC in the signal wavelength range. It is to be noted that the data presented here are not corrected for any AR coating or transmission losses of the crystal and mirror coatings.

The power scaling results of the PPKTP OPO are presented in Fig. 6. At a fixed temperature of $30^{\circ} \mathrm{C}$, and for a signal output coupling of $26 \%$, we extracted as much as $580 \mathrm{~mW}$ of average signal power at $765 \mathrm{~nm}$, together with $200 \mathrm{~mW}$ of idler at $1746 \mathrm{~nm}$, for an input pump power of $\sim 1.7 \mathrm{~W}$. The threshold of the OPO was $\sim 340 \mathrm{~mW}$ and a maximum pump depletion of $>60 \%$ was recorded. The slope efficiencies of the extracted signal and idler power are estimated to be $43 \%$ and $15 \%$, respectively. Similar measurements with identical output coupling, at an operating signal wavelength of $916 \mathrm{~nm}$, corresponding to an idler wavelength of $1269 \mathrm{~nm}$, resulted in an average signal power of 500 $\mathrm{mW}$ together with $300 \mathrm{~mW}$ of idler power, with slope efficiencies of $40 \%$ and $25 \%$, respectively, for an input pump power of $1.55 \mathrm{~W}$. This corresponds to a total average power of $800 \mathrm{~mW}$, representing an overall extraction efficiency of $\sim 52 \%$. The threshold of the OPO in this case was $\sim 280 \mathrm{~mW}$, while a pump depletion of $\sim 67 \%$ was recorded. No saturation was observed in either case, indicating the feasibility of further power scaling. However, the usable maximum pump power is currently limited by the material quality, as evident from Fig, 2 .

The simultaneously recorded long-term stability of the signal and idler power at a signal wavelength of $765 \mathrm{~nm}$, corresponding to an idler wavelength of $1746 \mathrm{~nm}$, is shown in Fig. 7(a). The signal power is measured using low power thermal detector (Ophir 3A-QUAD) with a response time of $\sim 2$ seconds, while the idler power is measured using another thermal detector (Ophir 10A-PPS), with a response time of $\sim 1$ second. The signal and idler power, recorded over a period of 1 hour while generating maximum output power, exhibit passive power stability better than $0.5 \% \mathrm{rms}$ and $0.8 \% \mathrm{rms}$, respectively. Figure 7 (b) shows the power stability of the pump at a power level of $\sim 2 \mathrm{~W}$, which is better than $0.8 \%$ rms over 1 hour.
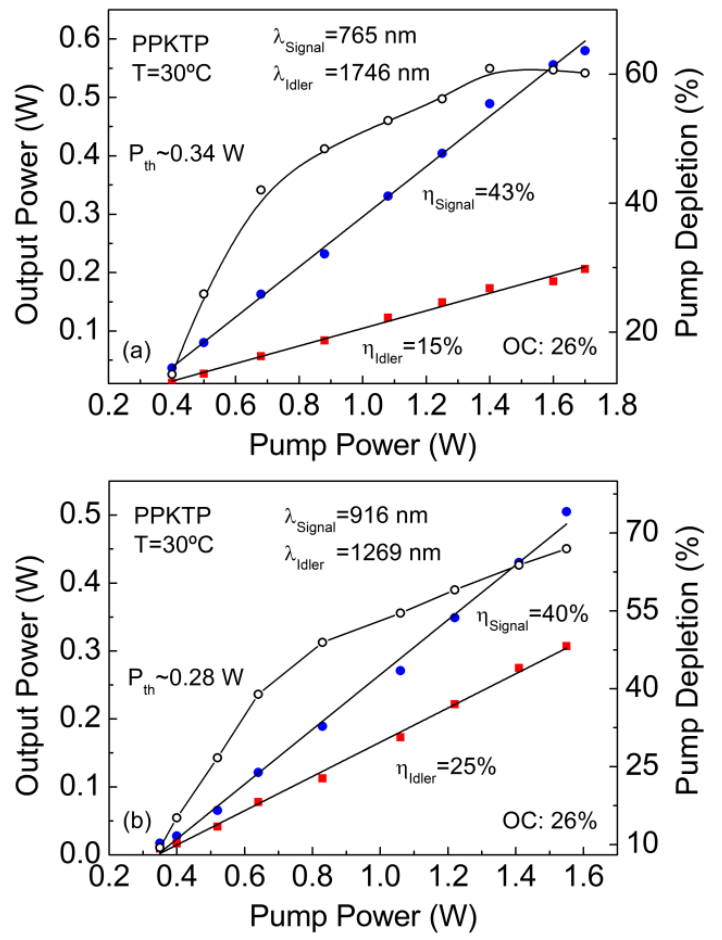

Fig. 6. Power scaling of the PPKTP OPO at an operating signal wavelength of (a) $765 \mathrm{~nm}$, and (b) $916 \mathrm{~nm}$.

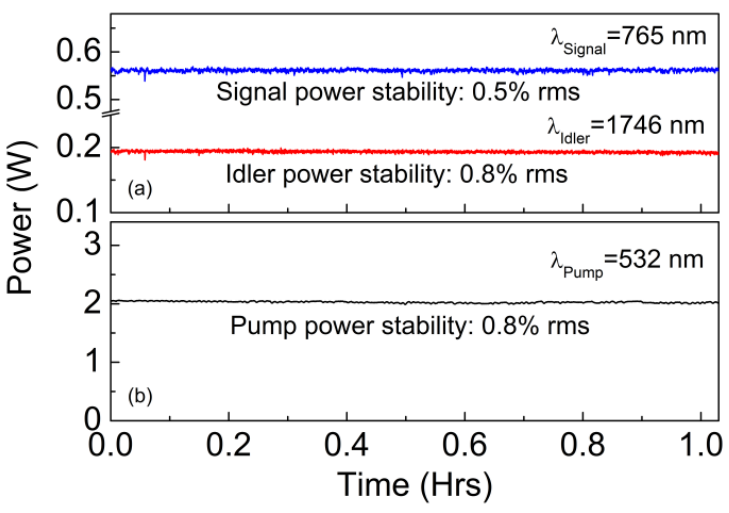

Fig. 7. Long-term power stability of the signal and idler together with the pump recorded over a period of 1 hour.
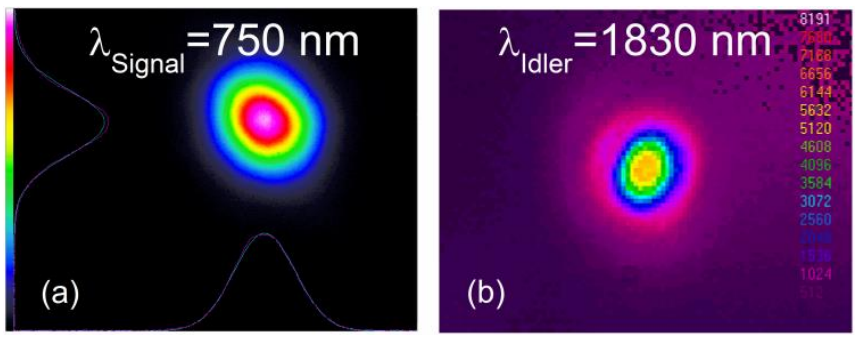

Fig. 8. Beam profiles of the extracted (a) signal, and (b) idler from the PPKTP OPO. 
The far-field energy distribution of the signal beam measured for a wavelength of $750 \mathrm{~nm}$ at the output of the OPO is shown in Fig. 8(a), with the corresponding idler beam profile shown in Fig. 8(b). The signal beam profile was recorded using a silicon-based CMOS camera and the near-infrared idler profile was recorded using a pyroelectric camera. Both beams are confirmed to exhibit TEMoo spatial profile with single-peak Gaussian distribution at maximum output power.

Further, we performed spectral and temporal characterization of the signal pulses from the PPKTP picosecond OPO at maximum output power. The long-term stability of the signal spectrum while operating

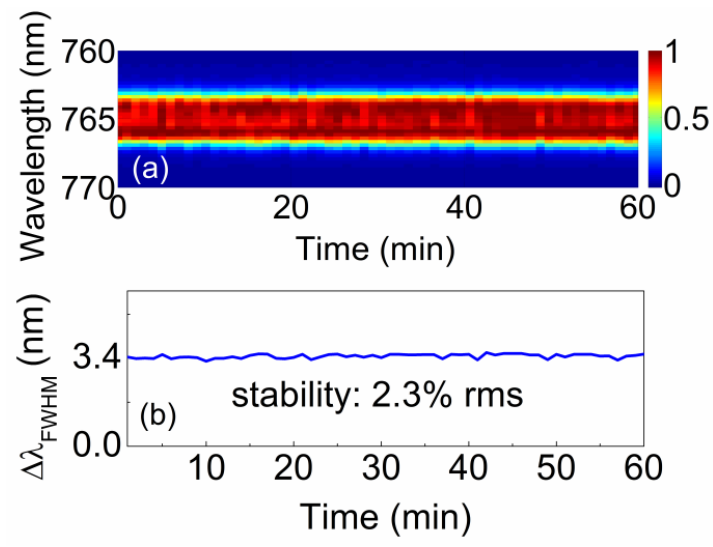

Fig. 9. Long-term (a) spectral stability, (b) FWHM bandwidth stability of the signal from the PPKTP OPO, recorded over a period of 1 hour. The color scale corresponds to the normalized spectral intensity.

at a central wavelength of $765 \mathrm{~nm}$ is shown in Fig. 9(a), indicating high spectral stability with a small double-peak feature in the spectrum. The corresponding FWHM signal bandwidth is stable to better than $2.3 \%$ rms over 1 hour at about $3.4 \mathrm{~nm}$, as shown in Fig. 9(b). Temporal characterization of the signal pulses from the OPO was performed by using home-made autocorrelator based on two-photon detector.

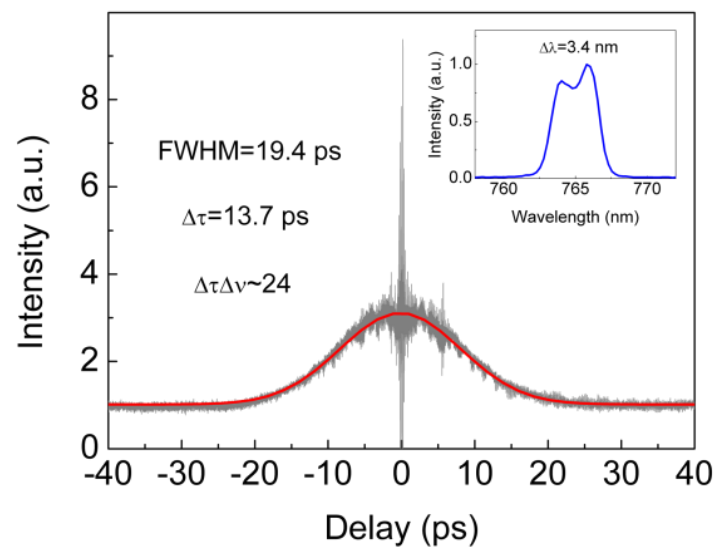

Fig. 10. Typical interferometric autocorrelation trace of the signal pulses extracted from the PPKTP OPO. Inset: Corresponding signal spectrum center at $765 \mathrm{~nm}$.

Figure 10 shows the typical interferometric autocorrelation trace, resulting in a FWHM pulse width of 19.4 ps, corresponding to a Gaussian pulse duration of 13.7 ps. The corresponding spectrum, with FWHM bandwidth of $3.4 \mathrm{~nm}$ centered at $765 \mathrm{~nm}$, is shown in the inset of Fig. 10. These measurements result in a time-bandwidth product of $\Delta \tau \Delta v \sim 24$ in the absence of dispersion compensation, compared to a time-bandwidth product of $\Delta \tau \Delta v \sim 10.3$ for the pump. Implementing intracavity dispersion compensation could further improve the timebandwidth product of the generated output pulses from the OPO.

In conclusion, we have demonstrated a stable picosecond OPO at 79.5 MHz based on PPKTP with a fan-out grating design, pumped by an Yb-fiber-based green source at $532 \mathrm{~nm}$, and widely tunable in the near-infrared close to room temperature. The OPO is continuously tunable over 726-955 nm in the signal and 1201-1998 nm in the idler, by grating tuning through mechanical translation of the fanned PPKTP crystal. The OPO provides $>400 \mathrm{~mW}$ of average power in the signal and $>100 \mathrm{~mW}$ in the idler over the entire tuning range for an input pump power of $\sim 1.6 \mathrm{~W}$, with a maximum of $580 \mathrm{~mW}$ in the signal at $765 \mathrm{~nm}$ and $300 \mathrm{~mW}$ in the idler at $1338 \mathrm{~nm}$. The extracted signal and idler from the OPO exhibit excellent passive power stability better than $0.5 \% \mathrm{rms}$ and $0.8 \% \mathrm{rms}$, respectively, over 1 hour, in high beam quality with TEM 00 mode profile. In the absence of dispersion control, the signal pulses extracted from the OPO have Gaussian temporal duration of 13.2 ps measured at $765 \mathrm{~nm}$ with an FWHM bandwidth of $3.4 \mathrm{~nm}$, resulting in a time-bandwidth product of $\Delta \tau \Delta v \sim 24$. The signal spectrum has a FWHM bandwidth stability of $2.3 \%$ rms measured at $765 \mathrm{~nm}$. Measurements of the green pump transmission in the PPKTP crystal have been performed, placing a limitation to power scaling of the OPO beyond $2.4 \mathrm{~W}$ of average pump power due to bulk damage in the material, with the damage mechanism identified as dependent on the average power rather than intensity of the green pump radiation. The overall extraction efficiency of the present device could potentially be improved by optimizing the output coupling at the current pumping level [6].

Funding sources and acknowledgments. We acknowledge support from the Ministry of Economy and Competitiveness (MINECO), Spain (project OPTEX, TEC2012-37853), Generalitat de Catalunya (ACCIÓ, project VALTEC13-1-0003), European Office of Aerospace Research and Development (EOARD) (grant FA8655-12-12128), and the European Commission (project Mid-Tech, H2020MSCA-ITN-2014), and Generalitat Catalunya (AGAUR, project SGR 2014-2016).

\section{References}

1. F. Ganikhanov, S. Carrasco, X. S. Xie, M. Katz, W. Seitz, and D. Kopf, Opt. Lett. 31, 1292 (2006).

2. M. Jurna, J. P. Korterik, H. L. Offerhaus, and C. Otto, Appl. Phys. Lett. 89, 251116 (2006).

3. C. W. Freudiger, W. Min, B. G. Saar, S. Lu, G. R. Holtom, C. He, J. C. Tsai, J. X. Kang, X. S. Xie, Science 322, 1857 (2008).

4. S Chaitanya Kumar and M Ebrahim-Zadeh, Laser Phys. 24, 025401 (2014).

5. M. Ebrahim-Zadeh and S. Chaitanya Kumar, IEEE J. Sel. Top. Quantum Electron. 20, 7600519 (2014).

6. S. Chaitanya Kumar, A. Esteban-Martin, and M. Ebrahim-Zadeh, Opt. Lett. 36, 1068 (2011).

7. P. E. Powers, T. J. Kulp, and S. E. Bisson, Opt. Lett. 23, 159-161 (1998).

8. M. Ebrahimzadeh, G. P. A. Malcolm, and A. I. Ferguson, Opt. Lett. 17, 183 (1992).

9. Ch. Grasser, D. Wang, R. Beigang, and R. Wallenstein, J. Opt. Soc. Am. B 10, 2218 (1993).

10. S. Chaitanya Kumar and M. Ebrahim-Zadeh, Opt. Lett. 38, 5349 (2013).

11. F. Kienle, D. Lin, S. Alam, H. S. S. Hung, C. B. E. Gawith, H. E. Major, D. J. Richardson, and D. P. Shepherd, J. Opt. Soc. Am. B 29, 144 (2012).

12. S. Chaitanya Kumar, E. Sanchez Bautista, and M. Ebrahim-Zadeh, Opt. Lett. 40, 403 (2015).

13. G. D. Boyd and D. A. Kleinman, J. Appl. Phys. 39, 3597 (1968).

14. H. Li, F. Zhou, X. Zhang, and W. Ji, Opt. Comm. 144, 75 (1997).

15. R. DeSalvo, A. A. Said, D. J. Hagan, E. W. Van Stryland, and M. SheikBahae, IEEE J. Quantum Electron. 32(8), 1324-1333 (1996).

16. S. Chaitanya Kumar, G. K. Samanta, and M. Ebrahim-Zadeh, Opt. Express 17, 13711 (2009).

17. G. K. Samanta, S. Chaitanya Kumar, M. Mathew, C. Canalias, V. Pasiskevicius, F. Laurell, and M. Ebrahim-Zadeh, Opt. Lett. 33, 2955 (2008)

18. K. Kato and E. Takaoka, Appl. Opt. 41, 5040 (2002).

19. W. Wiechmann, T. Fukui, H. Masuda, and S. Kubota, Opt. Lett.18, 1208 (1993). 